\title{
Energy Resolution of Modern EDS Spectrometers: Is the current ISO standard definition outdated?
}

\author{
Ralf Terborg $^{1}$ and Meiken Falke ${ }^{1}$ \\ ${ }^{1}$ Bruker Nano GmbH, Am Studio 2D, 12489 Berlin, Germany
}

It is important to define characteristic parameters for energy dispersive X-ray spectrometers (EDS) in order to compare their performance. The ISO 15632 standard [1] defines various types of specifications, e.g. intensity ratios of certain $\mathrm{L}$ and $\mathrm{K}$ lines ( $\mathrm{L} / \mathrm{K}$ ratio) or the peak-to-background ratio.

In the mentioned standard special interest is put into the measurement of the line widths (full-widthat-half-maximum, FWHM) to determine the energy resolution of the spectrometer.

The method defined in that standard is a simple procedure: An EDX spectrum of a specific sample should be recorded, e.g. Fe55 or manganese, CaF2, carbon, but also an EDS-TM001 or EDS-TM002 [2] sample can be used. After a linear background subtraction the channel with the highest intensity (peak center) needs to be found. Then the two neighboring channels on each side of the peak (lowand high-energy side) with counts just above and below half of the highest peak intensity channel should be selected. After a linear interpolation of the two channel pairs the (fractional) number of channels, multiplied by the channel widths gives the energy resolution of the line, Fig. 1.

While this procedure can be easily completed with a piece of paper and a pencil, it is just an estimation. Additionally, the procedure originates from the first edition of this standard from 2002. At this time the work was focused on $\mathrm{Si}(\mathrm{Li})$ detectors with energy resolution around $130 \mathrm{eV}$ for $\mathrm{Mn}$ $\mathrm{K} \alpha$ and around $60 \mathrm{eV}$ for $\mathrm{C}-\mathrm{K}$ lines. Since then silicon drift detectors (SDDs) have become the standard detector type, which have much better energy resolutions down to $121 \mathrm{eV}$ and $38 \mathrm{eV}$ for $\mathrm{Mn}$ $\mathrm{K} \alpha$ and $\mathrm{C}-\mathrm{K}$, respectively which is very close to the theoretical limit of about $118 \mathrm{eV}$ for $\mathrm{Mn}-\mathrm{K} \alpha$ and offers much better performance in the low energy region. Furthermore, the standard seems to focus on spectra with a channel width of $10 \mathrm{eV}$ although spectrometer systems with smaller channel widths, e.g. $2.5 \mathrm{eV}$, are meanwhile available.

In order to test if the proposed method can still be applied to SDDs with high energy resolution 130 spectra of the EDS-TM002 reference material [2,3,4] have been recorded with a Mn-K $\alpha$ peak intensity of around 40000 counts using an XFlash 6110 spectrometer. The Mn-K $\alpha$ peak has been evaluated according to the method proposed in the ISO standard and also with other peak fitting methods using different types of background correction, Fig. 2. The determination using the ISO standard method shows large fluctuations of around $+/-5 \mathrm{eV}$ and mostly underestimates the energy resolution while other peak fitting algorithms show a better agreement.

The results will be further compared and discussed.

\section{References:}

[1] ISO 15632:2012

[2] M Alvisi et al., Micosc. Microanal. 12 (2006) p. 406

[3] http://www.rm-certificates.bam.de/en/certificates/layered_and_surface_materials/index.htm

[4] V-D Hodoroaba, M Procop, Microsc. Microanal. 20 (2014) p. 1556 


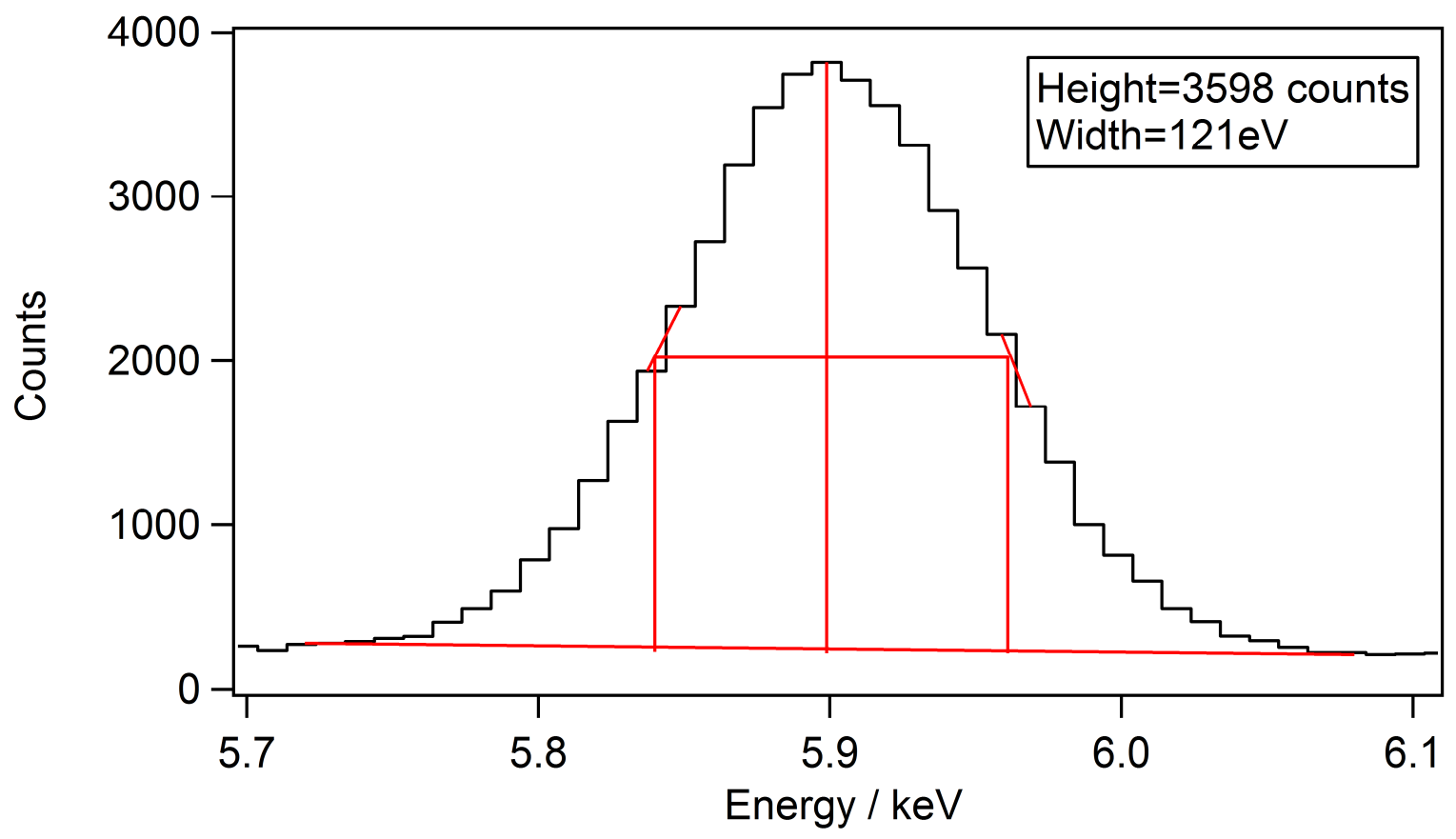

Fig. 1. Manganese Ka peak of a spectrum of an EDS-TM002 sample showing height and width of the peak.

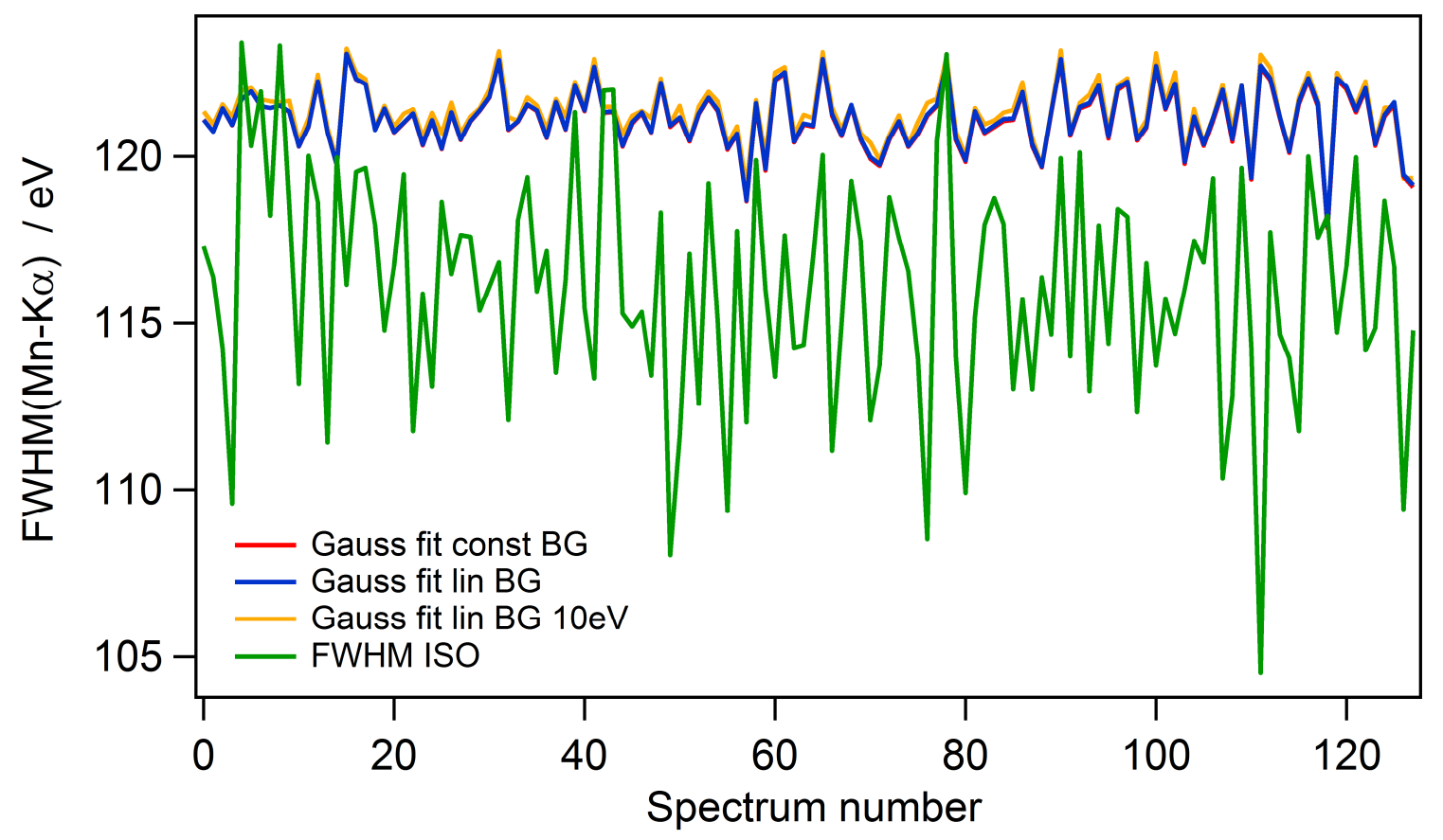

Fig. 2. Plot of the determined energy resolution of test spectra recorded with an XFlash 6110 SDD using Gaussian peak fits with different background correction and the method proposed in the ISO standard. 